Archives de sciences sociales des religions

148 | octobre-décembre 2009

Bulletin Bibliographique

\title{
Sacrifice, anthropologie, économie
}

\section{Tiina Arppe}

\section{(2) OpenEdition}

Journals

Édition électronique

URL : http://journals.openedition.org/assr/21475

DOI : $10.4000 /$ assr. 21475

ISSN : $1777-5825$

Éditeur

Éditions de l'EHESS

Édition imprimée

Date de publication : 31 décembre 2009

Pagination : 45-54

ISBN : 978-2-7132-2218-4

ISSN : 0335-5985

Référence électronique

Tiina Arppe, «Sacrifice, anthropologie, économie », Archives de sciences sociales des religions [En ligne], 148 | octobre-décembre 2009, mis en ligne le 01 janvier 2013, consulté le 02 mai 2019. URL : http:// journals.openedition.org/assr/21475; DOI : 10.4000/assr.21475 


\section{Tiina Arppe}

\section{Sacrifice, anthropologie, économie}

S'il fallait nommer une seule thématique qui traverse le grand livre de Camille Tarot, Le Symbolique et le sacré, ce serait sans doute le rapport entre la violence et le sacré - thématique girardienne par excellence. La violence constitue un complément nécessaire à la théorie durkheimienne de la religion, quoique "si Durkheim n'a pas vu la violence au sens girardien, il a vu l'effervescence » (Tarot, 2008a, p. 661). Dans le projet que se propose Tarot - la construction d'un « type idéal » de la religion, assez universel pour pouvoir subsumer tous les cas empiriques ou réels -, cette interprétation a sûrement son rôle à jouer, mais elle ne doit pas nous faire négliger les différences entre les deux théories. Dans ce qui suit nous présenterons d'abord quelques considérations concernant les anthropologies respectives de Girard et de Durkheim et la question de l'évolution, pour rebondir ensuite sur le problème de l'économie affective derrière les théories. Nous finirons par quelques remarques sur le rapport de ces questions au modèle de la religion que nous propose Tarot.

\section{La religion et l'ambivalence du sacré - la représentation vs la projection}

Si on juxtapose la théorie de la religion de Girard à celle de Durkheim, on peut effectivement dire - comme le fait Tarot - qu'elle la complète et qu'elle met en avant la violence que Durkheim n'a pas vue. C'est à travers cette violence, justement, que Girard veut regarder aussi bien la religion que la société. Au niveau théorique, sa solution consiste, en gros, à renverser l'interprétation durkheimienne de la religion en partant du sacré impur (que Durkheim a vu, mais d'une certaine manière neutralisé en mettant le sacré pur - ou le "sacré du respect »- au premier rang) et de réduire l'essence impure du sacré à un rite unique, nommément le sacrifice. Ce que Durkheim a ignoré, selon Girard, c'est l'identité de la religion et du sacrifice - le fait que la religion constitue un moyen rituel pour canaliser la violence collective, oscillant à l'état diffus, sur un objet " extérieur ", dont la destruction permet de la figer en "force " transcendantale (et bénéfique), à savoir le sacré (Girard, 2007a : 677). 
Pour Girard, l'impureté du sacré et sa dangereuse contagiosité - que Durkheim explique par la fameuse "ambivalence du sacré ", idée empruntée à Robertson Smith - ne sont que l'expression de la violence qui est la vraie essence du sacré. Bien qu'il ait parfaitement compris la fonction de la religion, Durkheim s'est donc trompé en pensant que l'objet de la religion (le sacré) était en réalité une représentation collective de la société et de sa «force ». Pour Girard, le sacré n'est pas une représentation, mais une projection collective par laquelle la communauté expulse de soi la violence engendrée par le désir (mimétique) humain. Or c'est ici qu'on tombe sur le premier écart significatif entre les deux théories, car entre la représentation et la projection il y a une différence essentielle : le refoulement - non pas l'interdit social (que Durkheim explique par le sentiment du respect suscité par le sacré « majestueux », i.e. la société, auprès du croyant), mais la répulsion intrapsychique, soit individuelle soit collective. Si l'on suit la définition psychanalytique de la projection, c'est une "opération par laquelle le sujet expulse de soi et localise dans l'autre, personne ou chose, des qualités, des sentiments, des désirs, voire des "objets", qu'il méconnaît ou refuse en lui » (Laplanche, Pontalis, 2002 : 344 sq.).

Chez Durkheim, c'est précisément cet élément qui manque. Le rite qu'il utilise pour expliquer l'ambivalence du sacré dans Les Formes élémentaires de la vie religieuse n'est pas le sacrifice mais les rites dits «piaculaires ». Selon Durkheim, les forces malfaisantes, c'est-à-dire le sacré impur, sont en fait un produit de ces rites : elles ne sont que des états collectifs objectivés. Les diverses manifestations de l'angoisse dans le rite restituent au groupe l'énergie que les événements malheureux (la mort, par exemple) menaçaient de lui soustraire, et lui permettent de se ressaisir. Ainsi, le groupe n'a pas besoin de refuser ou d'expulser ses propres sentiments négatifs mais a, au contraire, intérêt à les intensifier (ce qu'il fait, effectivement), car «toute communion des consciences (...) rehausse la vitalité sociale (...) En fait, quand le sentiment social est froissé douloureusement, il réagit avec plus de force qu'à l'ordinaire (...) Ce surcroît d'énergie efface d'autant plus complètement les effets du désemparement qui s'était produit à l'origine (...) » (Durkheim, 1990 : 544).

C'est pourquoi le lieu «par excellence » pour la transfiguration et la « fonctionnalisation " des affects négatifs dans la théorie durkheimienne n'est pas le sacrifice, mais les rites piaculaires. Il est vrai que Durkheim a aussi une théorie sur le transfert des sentiments, mais elle est également d'un caractère tout à fait positif. Cette théorie est présentée dans le contexte d'une scène d'origine hypothétique sur l'aube de la culture humaine qui, selon Durkheim, est à chercher dans les états d'effervescence intense. (ibid.: 313 sqq.). Pour expliquer comment la force de la société en vient à être associée à un emblème, Durkheim s'appuie sur la « loi connue » du transfert des sentiments au symbole qui représente l'objet original de ces sentiments. Comme la société est une entité trop abstraite, les sentiments qu'elle suscite sont transférés sur un signe qui prend sa place. Ainsi 
s'explique le statut particulier du totem, associé à l'état de surexcitation provoqué par le rituel. D'autre part, il y a un transfert proprement "mimétique » qui se produit dans la foule ("l'impulsion initiale va ainsi s'amplifiant à mesure qu'elle se répercute»), mais c'est l'épuisement physique et mental qui y met fin, et non un meurtre ou un sacrifice, comme dans le modèle girardien. En résumé, l'ambiance générale dans la «scène d'origine » que décrit Durkheim est excitée, mais positive : "Ce qui est à la racine du totémisme, ce sont, en définitive, des sentiments de joyeuse confiance plus que de terreur et de compression» (ibid.: 320-321). Pas de refoulement donc, car rien à refouler. Pas de projection directe de l'affect sur la victime (autrui), mais son transfert médiatisé sur le totem représentant le "grand Autre» (la société).

Tandis que dans la scène d'origine durkheimienne on chante et on danse, dans celle de Girard, on tue. La concurrence mimétique aboutit à un meurtre, et le corps inerte de la victime constitue le premier signifiant, la première différence significative qui met fin à la violence indifférenciée. Pendant les centaines de milliers d'années qui suivirent, le rituel émissaire - fondé sur ce premier lynchage - aboutit peu à peu à la naissance de la culture et de la langue humaines. Au cours de cette période, une nouvelle forme d'extériorisation et de maîtrise de la violence est née ; forme dans laquelle les mécanismes instinctifs de défense sont remplacés par des interdits et des rites, c'est-à-dire par des mécanismes culturels. L'efficacité inouie de ces derniers contribue à renforcer la tendance mimétique et conduit par là au développement de l'intelligence caractéristique de l'homme (Girard, 2007b : 815-819). Mais la condition indispensable pour le développement des mécanismes culturels de défense, et pour le fonctionnement $\mathrm{du}$ modèle girardien, n'est rien d'autre que l'expulsion affective violente et sa méconnaissance consécutive. Ainsi, pour Girard, la fameuse "ambivalence du sacré » n'est qu'un reflet du caractère " pharmacologique » de la violence, c'està-dire de son statut de "pharmakos" ou de poison qui guérit (Derrida, 1972). La violence impure, engendrée par le désir mimétique, est la violence non maîtrisée et contagieuse dont on ne peut se protéger que par la violence rituellement " purifiée » (le mécanisme émissaire). La distinction entre le pur et l'impur est vitale pour Girard, car elle constitue la condition sine qua non de la canalisation sacrificielle de la violence - la confusion entre les deux entraînerait la société entière dans le cercle vicieux de la violence destructrice.

\section{L'anthropologie durkheimienne et la question du désir}

Or, ce même type d'argument «hobbesien », soulignant les effets néfastes des passions déchaînées, se trouve également chez Durkheim, mais dans un contexte plus "moderne ", celui du suicide. C'est surtout dans Le Suicide que Durkheim met en avant le côté socialement dangereux de l'affectivité humaine, et le besoin de le régler de manière collective afin d'éviter un état d'anomie 
généralisé. Dans le même contexte, il présente une théorie anthropologique sur la nature du désir humain. Tandis que pour Girard la tendance mimétique est simplement plus forte chez l'homme que chez les autres animaux, pour Durkheim, il y a une différence fondamentale (qualitative) entre les deux types de désir : le désir humain est, dès le début, beaucoup plus «spirituel » que le besoin animal. Dans la théorie durkheimienne, c'est la réflexion qui pousse l'homme à désirer toujours plus et qui fait que la plupart de ses besoins ne sont pas directement sous la dépendance du corps. Toutefois, Durkheim ne dit pas explicitement comment et quand se produit ce type de réflexion chez l'homme; il affirme seulement que c'est « l'éveil de la conscience » qui rompt l'équilibre, naturel chez l'animal, entre les besoins et les moyens pour les satisfaire (Durkheim, 1960 : 275).

La nature potentiellement illimitée du désir humain est une caractéristique spécifique de l'espèce qui le distingue, justement, de celui des autres créatures vivantes. Comme le désir de l'homme ne connaît pas de limite naturelle, le frein nécessaire pour calmer cette "soif inextinguible» ne peut être trouvé dans la constitution psychologique ou organique de l'individu. Abandonné à lui-même, l'individu est totalement à la merci de la logique naturellement " excessive » de la passion: "Par elle-même, abstraction faite de tout pouvoir extérieur qui la règle, notre sensibilité est un abîme sans fond que rien ne peut combler. " (ibid. : 273). C'est pourquoi il faut trouver une force extérieure à l'individu, une puissance régulatrice qui aurait le même rôle par rapport aux "besoins moraux " que l'organisme par rapport aux besoins physiques. Mais comme les individus doivent, en plus, reconnaître la légitimité de ce pouvoir régulateur, seule la société peut, selon Durkheim, remplir ce rôle : «Dans la mesure où les appétits ne sont pas automatiquement contenus par des mécanismes physiologiques, ils ne peuvent s'arrêter que devant une limite qu'ils reconnaissent comme juste. » (ibid. : 273) ${ }^{1}$.

Il est vrai que Durkheim parle ici surtout de la régulation des passions dans la société moderne. Cependant, et même si dans la société archaïque l'autorité joue un rôle beaucoup plus important comme source de légitimité, la situation

1. Ici, Durkheim se situe d'une certaine manière entre la tradition de la théorie politique des XVII ${ }^{\mathrm{e}}$ et XVIII ${ }^{\mathrm{e}}$ siècles, qui essaye de contrôler les passions humaines dans le registre du droit, et un nouveau discours qui est en train de se former à la fin du XIX siècle et qui voit l'affectivité humaine comme un champ d'énergie objectivable et mesurable, et donc cherche le contrôle dans le registre du fait (cette distinction des registres vient de Stéphane Corbin qui l'utilise dans son excellente étude sur Rousseau, 2003). D’un côté, Durkheim continue à chercher pour les passions un moyen régulateur que les sujets de la domination pourraient vivre comme juste et auquel ils pourraient, par conséquent, se soumettre pour ainsi dire "spontanément "; d'un autre côté, il affirme que la « force » ou la puissance en question ressemble aux forces physiques, aux courants électriques qui, malgré leur caractère invisible, sont réels et produisent des effets tangibles et mesurables. Dans Le Suicide, «la société » est un mélange de ces deux courants de pensée : une force physico-morale, à laquelle s'ajoute plus tard (surtout dans les Formes), la dimension symbolique. Sur le changement plus général qui s'est produit aux XVIII et XIX ${ }^{\mathrm{e}}$ siècles dans le discours concernant l'affectivité humaine, $c f$. Arppe, 2005, pp. 25-30. 
de base n'y est pas forcément différente : pour contrôler l'affectivité humaine, il faut une autorité morale, extérieure aux individus. Or, bien que chez Girard on puisse trouver le même type d'hypothèse sur le manque de «frein naturel » à la violence mimétique humaine, son raisonnement part d'un monisme ontologique résolu et se fonde sur le caractère " automatique » (naturel) du processus mimétique. Selon lui, chez les primates le processus est identique : si un grand singe en voit un autre tendre la main pour prendre un objet, il essaie presque automatiquement d'attraper le même objet. Tout le reste n'est qu'un voile métaphysique destiné à cacher le caractère concurrentiel de la mimesis aux acteurs eux-mêmes. La même chose vaut pour le premier acte de violence (constituant l'origine de la culture) qui est un résultat machinal, inévitable (et nullement «spontané ») de la logique propre de l'imitation : l'objet original du désir s'oublie peu à peu, au fur et à mesure que la quantité des concurrents augmente et que la concurrence se transforme en hostilité généralisée, détruisant les différences entre les participants. Mais le fondement naturel (le désir mimétique) qui produit son résultat de manière quasi automatique reste le même, et ce fondement réside dans la constitution biologique de l'espèce. Il n'y a donc, ici, pas de rupture foncière entre la nature et la culture, et surtout pas de holisme, générateur d'un nouveau type d'être sui generis : pas d' "hyperspiritualité » (médiatrice de la relation natureculture) si essentielle pour la théorie sociale de Durkheim ${ }^{2}$ - uniquement un mécanisme plus efficace, produit quasi automatique du surcroît de mimétisme lié à l'augmentation du cerveau humain, permettant l'extériorisation (et le contrôle) de la violence et correspondant par définition au seuil de l'hominisation (Girard, 2007b : 814) ${ }^{3}$.

\section{L'évolution, monisme, dualisme}

Ainsi, dans la théorie de Girard, le désir mimétique est plutôt un facteur de rapprochement que de séparation entre l'homme et d'autres espèces animales : cette tendance est seulement plus développée chez l'homme que chez les autres espèces. Dans la constellation girardienne, la première raison pour cela est à chercher dans la structure sociale animale : chez les animaux le conflit mimétique ne peut pas s'étendre parce que, dans le groupe, les individus se soumettent à la volonté d'un seul, le leader, qui attrape toujours pour lui les meilleurs morceaux,

2. Durkheim appelle « hyperspiritualité » le pouvoir qu'a la vie sociale d'élever les attributs de la vie psychique à une plus haute puissance de manière qu'ils constituent quelque chose "d'entièrement nouveau ": Durkheim, 1974, p. 49 ; pour une critique de ce concept, voir Arppe, 2005, note 20; Karsenti, 1997, 43.

3. "Au-delà d'un certain seuil de puissance mimétique, les sociétés animales deviennent impossibles. Ce seuil correspond donc au seuil d'apparition du mécanisme victimaire ; c'est le seuil de l'hominisation... » (op. cit. : on voit bien qu'il s'agit d'une définition pure et simple ici : le seuil de l'hominisation est définie comme le seuil d'apparition du mécanisme victimaire.) 
les femelles les plus fécondes, etc. (mais pourquoi en serait-il autrement dans les hordes humaines primitives, Girard ne le dit pas). D'un autre côté, ce qui manque chez l'homme c'est un frein naturel ou instinctuel à la violence : le développement des outils et des armes fait que l'homme n'est plus capable de contrôler sa violence, alors que chez les animaux les seules armes dans le combat sont les dents, les griffes et les membres (Girard, 2007b : 804-809).

Girard lie étroitement sa propre explication à l'évolution humaine (au processus d'hominisation), à la nature biologique de l'homme et à ses conditions de développement, qui situent l'homme fermement sur le même continuum ontologique que les autres espèces. Or, sur ce point, sa théorie n'est pas conforme à celle de Durkheim qui, lui, part d'un dualisme ontologique - modéré, il est vrai, mais assez net justement au niveau anthropologique. Comme on le sait, chez Durkheim l'homme se distingue de l'animal par sa double nature : d'un côté, il y a le corps avec ses désirs égoïstes, prisonnier de ses instincts naturels ; de l'autre côté, il y a l'esprit, la pensée conceptuelle et les règles morales qui sont tous, en dernière instance, d'origine sociale (Durkheim, 1970 : 314-332). Autrement dit, bien que la nature soit une, la nature humaine est double, et c'est justement par sa nature sociale que l'homme transcende sa nature biologique, donnée ${ }^{4}$.

C'est dans ce sens que, chez Durkheim, la société fait toujours violence aux désirs naturels de l'homme. Bien que la société fasse partie de la nature biologique et physique, accepter le monisme matérialiste serait refuser le caractère spécifique des faits sociaux, le réduire à la biologie. Comme le constate Tarot, la socialité de l'homme n'est pas unique au sens où il y a aussi des sociétés animales (des abeilles, des fourmis, des loups, etc.), mais elle est unique en son genre, en tant que socialité humaine (2008a : 78). Or, selon Tarot, c'est justement là qu'entre en scène la religion : comme elle est, de part en part, une institution sociale (on ne connaît pas de société humaine sans religion) et comme la socialité fait partie de la nature spécifique de l'homme, la question de la religion se trouve au cœur du problème touchant le caractère spécifique de l'espèce humaine. La religion et la nature sociale de l'homme sont ainsi en rapport étroit. Dans Les Formes, Durkheim offre sa propre solution à ce problème en faisant de la religion le point focal où l'homme se transcende ; dans lequel il "transfigure » sa nature biologique, en créant au-dessus d'elle un ordre social et symbolique ayant sa nature propre et obéissant à ses propres lois.

Or, il semble que c'est justement ce niveau social, ou plutôt sa théorisation plus élaborée, qui fait défaut à la théorie de Girard. Son regard est anthropologique d'une manière assez étroite. Ainsi, il propose, par exemple, de penser l'hominisation à partir de la mimesis d'appropriation justement afin d'éviter une séparation trop abrupte entre la nature et la culture : "Nous voyons bien que

4. Voir aussi le livre précédent de Tarot qui a présenté une analyse perspicace du rapport de Durkheim à l'évolutionnisme (1999, p. 70 sq.). 
les paliers d'évolution biologique sont [chez l'homme] trop rapides pour ne pas impliquer des éléments culturels (...) le volume du cerveau s'accroît beaucoup trop vite pour les processus normaux de l'évolution biologique ». Mais, à part quelques observations, d'une nature plutôt générale, il ne théorise pas la manière dont la société intervient dans cette transition, ni d'ailleurs le processus historique qui a fait de nous ce que nous sommes. De la nature on passe directement à la culture, sans rupture ni médiation, car le mécanisme mimétique, enraciné dans la constitution anthropologique de l'homme, reste toujours identique à travers les différentes formations sociales et les vicissitudes de l'histoire. Bien que, dans le capitalisme avancé, la violence mimétique se canalise plus dans l'économie et le développement technologique que dans la religion, pour la théorie girardienne ces domaines ne sont qu'une manifestation « épiphénoménale » d'un désir naturel qui fonctionne comme le facteur quasi causal de l'explication: "Nous pouvons concevoir l'hominisation comme une série de paliers qui permettent de domestiquer des intensités mimétiques toujours croissantes (...) » (2007 : 815). Seulement, à la place de «l'hominisation » on pourrait tout aussi bien mettre « la culture humaine». C'est ce qui rend la théorie girardienne si monotone.

\section{Le sacrifice, la religion et l'économie}

Toutefois, entre la théorie de Girard et celle de Durkheim, il existe des ressemblances à partir de leur tentative de trouver une explication universelle à la religion. Le point principal qui les unit est ce que je voudrais appeler une « économie fonctionnaliste de l'affectivité ». Quoique l'ambiance de la scène d'origine chez Girard soit très loin de l'euphorie joyeuse des primitifs de Durkheim, sa théorie sur la naissance de la culture est structurellement assez proche du modèle durkheimien : pour les deux, la canalisation de l'affectivité humaine de manière productive constitue le moment fondateur de la culture, c'est-à-dire son détachement de la nature. Après cela, l'affect s'attache durablement à un signifiant (chez Girard la victime, chez Durkheim le totem) qui commence à fonctionner comme son signe; ce qui entraîne la naissance de la langue. À cet égard, la vision de Girard est étonnamment proche de Durkheim, jusqu'au vocabulaire choisi : "On voit très bien que le langage articulé (...) doit se constituer, lui aussi, à partir du rite, à partir des hurlements et des cris qui accompagnent la crise mimétique, et que le rite doit reproduire eux aussi, puisqu'ils précèdent et peut-être conditionnent l'immolation salvatrice (...) [D]ans la pratique rituelle, autour de la victime, ces cris d'abord inarticulés commencent à se rythmer et s'ordonner comme les gestes de la danse (...)»(Girard, 2007b : 824) $)^{5}$. Au commencement, il y a un affect homogène duquel découle toute la diversité des cultures et des

5. À comparer avec la manière dont Durkheim décrit la naissance du symbolisme (1990, pp. 329-330). 
religions. Dans la théorie girardienne, c'est le premier lynchage «spontané » qui représente le "big-bang » générateur des différences; dans la théorie durkheimienne, par contre, il n'y a pas un tel événement fondateur, le système des différences se développant petit à petit, "dans ces milieux sociaux effervescents » (Durkheim, $1990: 313$ ).

Le même problème " économique » unit donc les anthropologies de Durkheim et de Girard : comment canaliser l'affectivité humaine de façon productive, c'està-dire comment domestiquer les impulsions « naturelles » de l'homme, potentiellement dangereuses, de telle manière que d'une force destructrice elles deviennent une force civilisatrice ? Pour les deux, la solution à ce problème déclenche la culture humaine ${ }^{6}$. Mais comme le fait remarquer Tarot, c'est le côté symbolique qui fait défaut à Girard - ou, pour suivre la formulation de François Gauthier, du sacré durkheimien Girard ne conserve que la composante énergétique (l'autre composante étant dans le modèle de Gauthier le "topique », plutôt du côté du symbole et de la structure) (2008).

C'est peut-être surtout cette vocation « universaliste » qui unit Tarot à Durkheim aussi bien qu'à Girard. Son ambition est de construire un modèle universel de la religion, mais sans les présuppositions causalistes «lourdes » de ces derniers. Le modèle que vise Tarot est plus proche du «type-idéal » wébérien, c'est-à-dire d'un outil heuristique de description ou de classification qu'une «essence » anthropologique réelle. C'est pourquoi il ne partage pas nécessairement l'économie affective fonctionnaliste décrite ci-dessus ${ }^{7}$, ni la prémisse " réaliste » de la théorie de Girard, selon laquelle au commencement il y aurait un meurtre réel. "Cette religion pure par en bas n'est pas une réalité ou un objet empirique et historique, mais la résultante de la construction d'un modèle... » (Tarot, 2008 : 689). Et justement, comme il le spécifie lui-même plus tard, c'est ce qui fait sa différence avec Girard, pour qui cette construction n'est pas un modèle, une grille d'interprétation de la réalité, mais son explication directe. (Tarot, 2008b : 28).

À mon avis, Tarot apporte deux facteurs essentiels qui changent beaucoup sa configuration par rapport à Girard : l'histoire et le symbolique. Lorsqu'on commence à parler des « mécanismes universels ", c'est souvent l'un ou l'autre qui fait défaut. Or, pour Tarot, le mécanisme émissaire, bien qu'il existe, n'est pas nécessairement producteur de religion parce que, pour cela, il faut des circonstances

6. Cette problématique «hobbesienne » est à la racine de toute la théorie politique moderne, mais c'est la conception du mécanisme à même de contrôler la frénésie passionnelle et le domaine dans lequel est cherchée la solution qui distingue les théories entre elles ; sur la relation de Durkheim à cette tradition du droit naturel, voir Arppe, 2005, pp. 25 sq. ; sur le rapport Girard-Hobbes, voir Scubla, 2003, pp. 217 sq.

7. Tout au moins pas dans ses présuppositions ontologiques, quoique la maîtrise de la violence impure constitue bel et bien, pour lui aussi, l'une des fonctions principales du côté rituel de la religion. 
historiques et anthropologiques spécifiques. Or, le mécanisme émissaire n’est pas symbolique mais réel. Pour comprendre le niveau phénoménologique/historique, il faut le compléter par les faits, mais le résultat n'est pas donné à l'avance. Autrement dit, il semble que, pour comprendre le processus historique qui a fait une religion particulière, la "nature » anhistorique n’a jamais été suffisante. Le symbolique entre en jeu justement à ce point-là : « ... tout ceci n'est possible que parce qu'entre le récit et le réel, il y a nécessairement une mémoire ou se mixent l'imaginaire et le réel... » (Tarot, 2008b : 28). Le défaut de Girard, c'est de ne pas vouloir théoriser cette mémoire autrement que sous la forme d'une "méconnaissance » universelle. C’est pourtant dans ce même interstice que réside la possibilité de l'histoire, tout ce qui est du côté de la distanciation, du récit, de la mémoire individuelle ou collective, du travail symbolique qui ne nous rend jamais le réel comme tel.

Tiina ARPPE Académie de Finlande - Département de sociologie tiina.arppe@helsinki.fi

\section{Bibliographie}

Arppe Tiina, 2005, «Rousseau, Durkheim et la constitution affective du social », Revue d'Histoire des Sciences Humaines, 13, pp. 5-32.

Corbin Stéphane, 2003, Jean-Jacques Rousseau : l'autre socio-anthropologie politique. Thèse d'Université, Université de Basse-Normandie.

Derrida Jacques, 1972, "La pharmacie de Platon ", in Derrida J., La Dissémination, Paris, Seuil.

Durкheim Émile, [1897] 1960, Le Suicide, Paris, Presses Universitaires de France.

-, [1912] 1990, Les formes élémentaires de la vie religieuse, Paris, Presses Universitaires de France.

-, [1914] 1970, "Le dualisme de la nature humaine et ses conditions sociales ", in Durkheim E., La Science sociale et l'action, Paris, Presses Universitaires de France.

-, [1898] 1974, "Représentations individuelles et représentations collectives ", in Durkheim E., Sociologie et philosophie, Paris, Presses Universitaires de France.

GAUTHIER François, 2008, "Enjeux pour une théorie de la religion au-delà du mirage girardien. À propos de Le symbolique et le sacré de Camille Tarot ». Revue du MAUSS en ligne, le 11 septembre 2008.

GIRARD René, 2007a, "La violence et le sacré ", in Girard R., De la violence à la divinité, Paris, Grasset, pp. 293-699.

-, [1978] 2007b, Des choses cachées depuis la fondation du monde, in Girard R., De la violence à la divinité, Paris, Grasset, pp. 701-1221.

KARSENTI Bruno, 1997, L'homme total. Sociologie, anthropologie et philosophie chez Marcel Mauss, Paris, Presses Universitaires de France.

Laplanche Jean, Pontalis Jean-Bertrand, [1967] 2002, Vocabulaire de la psychanalyse, Paris, Presses Universitaires de France. 
SCUBLA Lucien, 2003, "Roi sacré, victime sacrificielle et victime émissaire ", Revue du MAUSS, 22, "Qu'est-ce que le religieux ? ", pp. 197-221.

TARот Camille, 1999, De Durkheim à Mauss. L'invention du symbolique, Paris, La Découverte.

-, 2008a, Le symbolique et le sacré. Théories de la religion, Paris, La Découverte.

-, 2008b, "Religion, faut-il avoir peur, de qui et de quoi ? ", Revue du MAUSS en ligne, le 7 novembre 2008 . 